\title{
Open
}

\section{Underuse of Surveillance Colonoscopy in Patients at Increased Risk of Colorectal Cancer}

\author{
Caitlin C. Murphy, MPH${ }^{1}$, Carmen L. Lewis, MD, MPH ${ }^{2}$, Carol E. Golin, MD $^{3,4}$ and Robert S. Sandler, MD, MPH ${ }^{1,5}$
}

OBJECTIVES:

Colorectal cancer incidence and mortality have declined over the past two decades, and much of this improvement is attributed to increased use of screening. Approximately $25 \%$ of patients who undergo screening colonoscopy have premalignant adenomas that require removal and follow-up colonoscopy. However, there are few studies of the use of surveillance colonoscopy in increased risk patients with previous adenomas.

METHODS: $\quad$ We conducted a cross-sectional study to examine factors associated with underuse of surveillance colonoscopy among patients who are at increased risk for colorectal cancer. The study population consisted of patients with previously identified adenomatous polyps and who were due for follow-up colonoscopy. Patients were categorized as attenders $(n=100)$ or non-attenders $(n=104)$ on the basis of completion of follow-up colonoscopy. Telephone surveys assessed the use of surveillance colonoscopy across domains of predisposing patient characteristics, enabling factors, and patient need. Mutlivariable logistic regression was used to identify factors associated with screening completion.

RESULTS: $\quad$ Perceived barriers, perceived benefits, social deprivation, and cancer worry were associated with attendance at colonoscopy. Higher benefits (odds ratio (OR) $2.37,95 \%$ confidence interval $(\mathrm{CI}) 1.04-$ 5.41 ) and cancer worry (OR $1.73,95 \% \mathrm{Cl} 1.07-2.79)$ increased the odds of attendance at follow-up colonoscopy, whereas greater barriers (OR $0.49,95 \% \mathrm{Cl} 0.28-0.88$ ) and high social deprivation ( $\geq 2$; OR $0.09,95 \% \mathrm{CI} 0.01-0.76$ ) were associated with lower odds.

CONCLUSIONS: $\quad$ Our results suggest that multilevel factors contribute to the use of surveillance colonoscopy in higher risk populations, many of which are amenable to intervention. Interventions, such as patient navigation, may help facilitate appropriate use of surveillance colonoscopy.

Am J Gastroenterol 2015; 110:633-641; doi:10.1038/ajg.2014.344; published online 11 November 2014

\section{Background}

Although colorectal cancer remains the second leading cause of cancer death among men and women in the US, both the incidence and mortality have declined over the past two decades (1). A substantial proportion of this improvement has been attributed to an increased use of screening (2). Data from the National Health Interview Survey (NHIS) from 2000 to 2010 suggest that most of the increase in screening has been due to increased use of colonoscopy (3). An estimated 11-14 million colonoscopies are performed in the US annually $(4,5)$.
Approximately $25 \%$ of patients who undergo screening colonoscopy have premalignant adenomas that require removal and follow-up (i.e., surveillance) colonoscopy. These patients are considered to be at increased risk of colorectal cancer, and current guidelines recommend that they undergo a repeat exam in 3-5 years (6). However, there have been surprisingly few studies of surveillance colonoscopy in patients with previous adenomas. Underuse of surveillance colonoscopy remains an ongoing concern in this population (7).

Appropriate use of surveillance colonoscopy may ultimately improve quality and reduce the cost and burden of colorectal

${ }^{1}$ Department of Epidemiology, Gillings School of Public Health, The University of North Carolina at Chapel Hill, Chapel Hill, North Carolina, USA; ${ }^{2}$ Department of Medicine, Division of General Internal Medicine, University of Colorado, Boulder, Colorado, USA; ${ }^{3}$ Department of Medicine, School of Medicine, The University of North Carolina at Chapel Hill, Chapel Hill, North Carolina, USA; ${ }^{4}$ Department of Health Behavior, Gillings School of Public Health, The University of North Carolina at Chapel Hill, Chapel Hill, North Carolina, USA; ${ }^{5}$ Center for Gastrointestinal Biology and Disease, The University of North Carolina at Chapel Hill, Chapel Hill, North Carolina, USA. Correspondence: Robert S. Sandler, MD, MPH, Center for Gastrointestinal Biology and Disease The University of North Carolina at Chapel Hill, CB\# 7555, 4157 Bioinformatics Building Chapel Hill, Chapel Hill, North Carolina 27599-7555, USA. E-mail: rsandler@med.unc.edu 
cancer. To address this research need, we examined multilevel factors contributing to underuse of surveillance colonoscopy among patients who are at increased risk for colorectal cancer. Understanding patterns of and factors associated with surveillance colonoscopy use is an important first step toward identifying effective strategies to promote appropriate use of colonoscopy.

\section{Methods}

\section{Participants and procedures}

We conducted a cross-sectional study of factors associated with the use of surveillance colonoscopy among patients with a history of colorectal adenoma. Patients who underwent screening colonoscopy at the University of North Carolina (UNC) Hospitals (Chapel Hill, NC), with previously identified adenomatous polyps, and who were due for follow-up colonoscopy were eligible for the study. Endoscopy documentation software (ProVation ${ }^{\circledR}$ Medical, Minneapolis, MN) at UNC generates appointment letters that are sent to patients when the next colonoscopy should be scheduled. Letters are based on the interval recommended by the treating physician and entered into the database following the initial exam. Follow-up intervals are selected on the basis of the endoscopic findings and pathology reports. Patients who do not schedule an exam after receiving the appointment letter are sent up to two reminder letters. No further contact is made after both reminder letters are sent. To determine the use of surveillance colonoscopy, we generated lists of patients aged $\geq 30$ years who were sent an appointment letter (i.e., required a follow-up exam) during a consecutive 3-month period (July-September 2012). We defined "attenders" as patients who completed a follow-up colonoscopy. Completion of colonoscopy was measured by electronic medical record and later verified by patient self-report. "Non-attenders" were patients who did not schedule a follow-up exam after 90 days of receiving a second reminder letter or did not complete a colonoscopy. Both patients who did not schedule an appointment and patients who scheduled an appointment but did not arrive were considered "non-attenders."

We randomly sampled 340 potentially eligible participants from the total list of attenders and non-attenders $(n=446)$. Sampling probabilities were based on the distribution of age, sex, and race/ ethnicity of non-attenders. A research assistant reviewed the electronic medical record to exclude patients who were non-English speaking or with reasons for a follow-up exam other than adenoma (e.g., inflammatory bowel disease, cancer; $n=51$ ). The remaining eligible participants $(n=289)$ were then sent an invitation letter and brochure describing the study. The letter also included a telephone number to request additional information or to opt out of the study. Of the eligible participants, 64 (22.1\%) could not be contacted and $21(7.3 \%)$ refused. Participants with undeliverable letters were replaced one-for-one until we achieved our target number of 100 interviewed participants in each group (Figure 1).

Two weeks following any non-returned invitation letter (i.e., not returned because of wrong address or patient did not opt out), the research assistant called patients to describe the study and determine whether the patient was interested in participat-

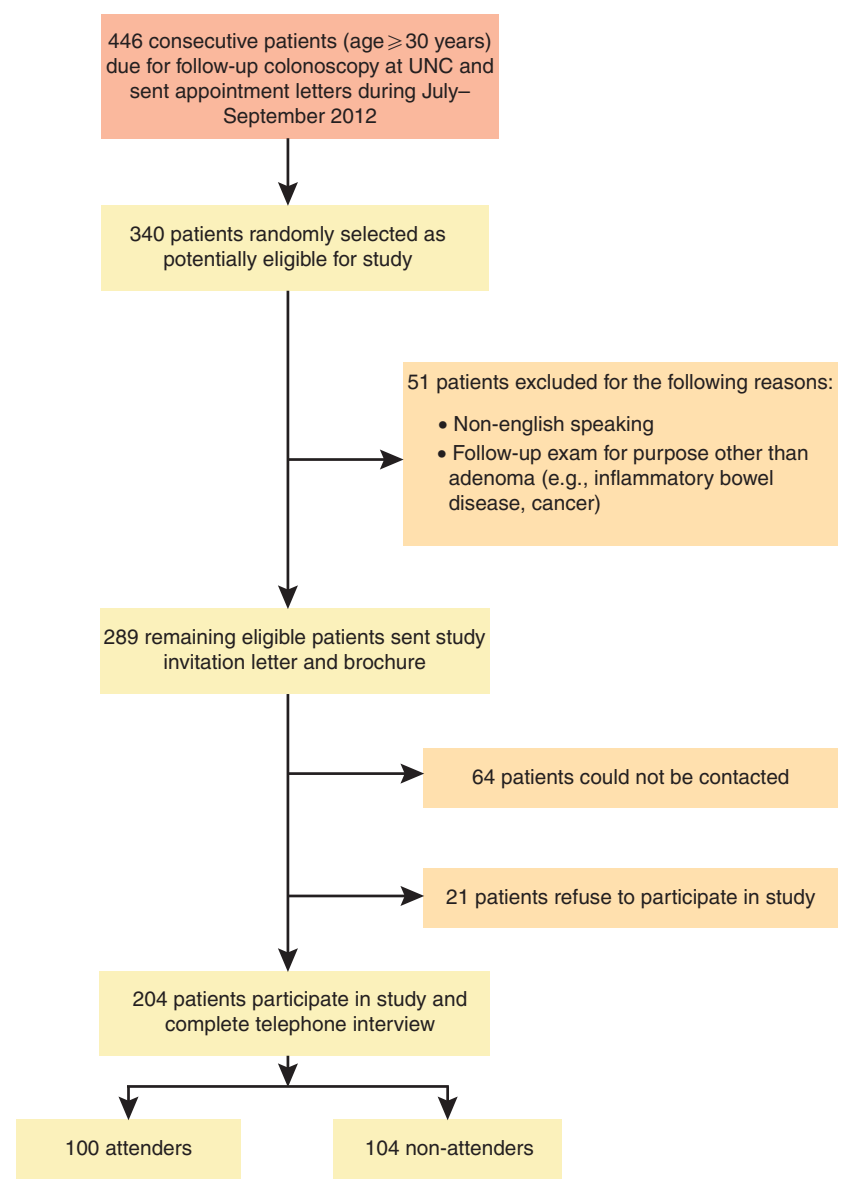

Figure 1. Process of participant selection into the study.

ing. Up to nine call attempts (with three voicemail messages) were made to reach the participants. Once a participant was reached, the research assistant explained the purpose of the study, verified receipt of the appointment letter ( $>80 \%$ of study participants recalled receiving the letter), and administered an interview survey to willing participants. The study invitation and survey were most often conducted in a single telephone call (median, 1; range, $1-4)$. Telephone calls were an average duration of $20.4 \mathrm{~min}$ (range 8-59 min). Participants were sent a \$20 gift card, as compensation for completing the survey.

\section{Measures}

We developed the telephone interview survey on the basis of a conceptual framework derived from Andersen's Behavioral Theory of HealthCare Utilization (8), which suggests that a patient's use of health services is a function of their predisposition to use services, factors that enable or impede use, and their need for care. All items and measures included in the telephone survey are listed in the Appendix 1.

Predisposing characteristics. Predisposing characteristics included demographic factors (e.g., age and sex), social structure (e.g., education, employment, race/ethnicity), and health beliefs 
(e.g., attitude, values, norms). Health beliefs were assessed by 20 items that measured psychosocial constructs related to colorectal cancer screening: perceived barriers, perceived benefits, and selfefficacy to be screened. Items and scales were adapted from colorectal cancer screening intervention trials (9-11) and have been validated in diverse settings (12-17). Perceived barriers measured negative aspects of screening ( 10 items, $\alpha=0.68$ ), and perceived benefits measured positive aspects ( 7 items, $\alpha=0.76$ ). Self-efficacy assessed a participant's confidence in their ability to perform certain aspects related to screening ( 4 items, $\alpha=0.77$ ). Barrier items were measured on a 5-point scale ranging from " 1 " for "strongly disagree" to " 5 " for "strongly agree", and benefits and self-efficacy items were measured on a 4-point scale ranging from "1" for "strongly disagree" to " 4 " for "strongly agree".

Enabling factors. Enabling factors must be present for a patient to effectively use health services (8). We assessed health insurance, insurance type, regular source of care (i.e., usual primary care physician), difficulty understanding the appointment letter, out-of-pocket costs, and social deprivation. Social deprivation was based on an index of socioeconomic deprivation developed by Power et al. (18) to predict colorectal cancer screening intention and attendance. The index combines dichotomous answers (i.e., yes/no) to questions on education, home ownership, and car ownership to create a scale from 0 (low deprivation) to 3 (high deprivation).

Patient need. Patient need factors considered how participants viewed their own health and functional state, as well as how they experience symptoms of illness, pain, and worries about their health. Health status variables included comorbidity, recent inpatient hospitalizations (within last year), smoking history, and functional status. Patient comorbidity was measured using an adapted version of the Charlson Comorbidity Index based on self-report (19). Functional status was measured using the selfreported Instrumental Activities of Daily Living scale (20) and a single 5-point item from the SF-12 ("How would you describe your general health for someone your age?”) (21). We also measured cancer worry and perceived susceptibility with previously validated scales adapted from colorectal cancer screening trials (12). Cancer worry measured negative effects related to the threat of colorectal cancer with four items $(\alpha=0.63)$ and perceived susceptibility measured subjective personal risk of colorectal cancer or polyps with three items $(\alpha=0.78)$. All items were measured on a 4-point scale, where higher scores correspond to higher levels of the variable being measured.

\section{Statistical analysis}

Pearson's $\chi^{2}$ or Fisher's exact tests were used to compare categorical characteristics of attenders and non-attenders, and Student's $t$-tests or Wilcoxon rank-sum tests were used to compare continuous characteristics.

To build a multivariable model, we first calculated Spearman's correlation coefficient among scales and covariates to assess collinearity of potential correlates. Participants' age and race were included as a priori correlates of attendance at follow-up colonoscopy. Other variables were selected if they were significantly associated with attendance in the univariable analysis $(P<0.25)$, were answered by most participants, and were not highly correlated with one another $(\rho<0.5)$. We also aimed for representation of covariates across the categories of predisposing characteristics, enabling factors, and patient need.

Using these criteria, we selected the following covariates to include in a multivariable logistic regression model: age, race, marital status, education, intention, barriers, benefits, self-efficacy, social deprivation, comorbidity, family history of colorectal cancer, previous inpatient hospitalizations, smoking status, self-rated health, functional status, cancer worry, and perceived susceptibility. Wald's selection of $P=0.10$ was then used to remove variables until a reduced model for the data was obtained. Associations between all variables in the reduced model and attendance at follow-up colonoscopy are reported as prevalence ORs and 95\% CIs.

Statistical analyses were conducted using SAS version 9.3 (SAS Institute, Cary, NC).

\section{Results}

Characteristics of the study population are shown in Table $\mathbf{1}$. Most participants were white/non-Hispanic (87.3\%), female (52.9\%), had a college degree or higher $(62.4 \%)$, were married or living with a partner $(70.9 \%)$, and retired $(46.3 \%)$. The mean age was 67.5 years (s.d. 9.48).

When building the multivariable model, greater perceived benefits, higher self-efficacy, cancer worry, and higher self-rated health were associated with higher odds of completing follow-up colonoscopy in the bivariate analysis. Greater perceived barriers, high social deprivation $(\geq 2)$, and one or more previous inpatient hospitalizations lowered the odds of screening completion (results not shown in table).

Perceived barriers, perceived benefits, social deprivation, and cancer worry remained statistically significant $(P<0.05)$ in the reduced model (Table 2). Higher perceived benefits (OR 2.37, 95\% CI 1.04-5.41) and cancer worry (OR 1.73, 95\% CI 1.072.79 ) increased the odds of attendance at follow-up colonoscopy, whereas greater perceived barriers (OR 0.49, 95\% CI 0.28-0.88) and high social deprivation ( $\geq 2$; OR $0.09,95 \%$ CI $0.01-0.76)$ were associated with lower odds.

Because perceived barriers emerged as an important factor related to attendance at surveillance colonoscopy, we further examined the mean score for each scale item among attenders and non-attenders. Non-attenders scored higher on the majority of perceived barriers (Table 3). Barriers related to cost, insurance coverage and co-pay, transportation, time, and care after colonoscopy appeared more salient for non-attenders.

\section{Discussion}

Our results suggest that there are multilevel factors that contribute to the use of surveillance colonoscopy in populations at 
Table 1. Characteristics of the study population by attendance at follow-up colonoscopy $(n=204)$

\begin{tabular}{|c|c|c|c|c|c|}
\hline Variable & \multicolumn{2}{|c|}{ Attenders $(n=100)$} & \multicolumn{2}{|c|}{ Non-attenders $(n=104)$} & $P$-value \\
\hline \multicolumn{6}{|l|}{ Predisposing characteristics } \\
\hline Age, range (mean, s.d.) & \multicolumn{2}{|c|}{ 49-97 $(67.8,9.09)$} & \multicolumn{2}{|c|}{ 51-92 (67.3, 9.88) } & 0.724 \\
\hline Sex & & & & & 0.269 \\
\hline Male & 51 & 53.1 & 45 & 46.9 & \\
\hline Race & & & & & 0.025 \\
\hline White & 90 & 50.6 & 88 & 49.4 & \\
\hline Non-white & 10 & 38.5 & 16 & 61.5 & \\
\hline Marital status & & & & & 0.074 \\
\hline High school or some college & 30 & 38.0 & 49 & 62.0 & \\
\hline College degree or higher & 69 & 56.1 & 54 & 43.9 & \\
\hline Employment & & & & & 0.565 \\
\hline Full-time/part-time & 43 & 53.1 & 38 & 46.9 & \\
\hline Retired & 44 & 46.8 & 50 & 53.2 & \\
\hline Not working & 12 & 42.9 & 16 & 57.1 & \\
\hline Intention & & & & & 0.211 \\
\hline Strongly agree/agree & 96 & 51.6 & 90 & 48.4 & \\
\hline Insurance type & & & & & 0.949 \\
\hline Medicare & 50 & 51.0 & 48 & 49.0 & \\
\hline Medicaid & 4 & 40.0 & 6 & 60.0 & \\
\hline Employer/union based & 38 & 49.4 & 39 & 50.7 & \\
\hline Private/other & 6 & 50.0 & 6 & 50.0 & \\
\hline Primary care physician & & & & & 0.369 \\
\hline Yes & 99 & 49.8 & 100 & 50.3 & \\
\hline No & 1 & 20.0 & 4 & 80.0 & \\
\hline Letter difficulty & & & & & 0.411 \\
\hline No difficulty understanding & 70 & 45.8 & 83 & 54.3 & \\
\hline At least some difficulty understanding & 6 & 35.3 & 11 & 64.7 & \\
\hline Out-of-pocket costs & & & & & 0.938 \\
\hline No & 33 & 50.8 & 32 & 49.2 & \\
\hline Yes & 56 & 51.4 & 53 & 48.6 & \\
\hline
\end{tabular}


Table 1. Continued

\begin{tabular}{|c|c|c|c|c|c|}
\hline Social deprivation index & & & & & 0.011 \\
\hline 0 & 84 & 53.2 & 74 & 46.8 & \\
\hline 1 & 14 & 42.4 & 19 & 57.6 & \\
\hline \multicolumn{6}{|l|}{ Patient need } \\
\hline \multicolumn{6}{|l|}{ Comorbidity } \\
\hline 1 & 27 & 40.3 & 40 & 59.7 & \\
\hline$\geq 2$ & 11 & 47.8 & 12 & 52.2 & \\
\hline \multicolumn{3}{|l|}{ Family history of CRC (1st degree relative) } & & & 0.223 \\
\hline No & 75 & 46.6 & 86 & 53.4 & \\
\hline Once or more & 9 & 28.1 & 23 & 71.9 & \\
\hline \multicolumn{3}{|l|}{ Smoker } & & & 0.147 \\
\hline Never/former & 92 & 50.8 & 89 & 49.2 & \\
\hline Current & 8 & 34.8 & 15 & 65.2 & \\
\hline \multicolumn{3}{|l|}{ Need help with daily activities } & & & 0.193 \\
\hline No & 95 & 50.5 & 93 & 49.5 & \\
\hline Yes & 5 & 31.3 & 11 & 68.8 & \\
\hline \multicolumn{3}{|l|}{ Difficulty walking 1/4 Mile } & & & 0.053 \\
\hline Not at all & 85 & 52.5 & 77 & 47.5 & \\
\hline
\end{tabular}

increased risk of colorectal cancer, many of which are psychosocial constructs amenable to intervention. Both cancer worry and higher perceived benefits were associated with completion of colonoscopy. The role of cancer worry is debated in the literature as to whether it motivates or impedes adherence to cancer screening behaviors (22), and previous work in colorectal cancer screening has found inconsistent associations between cancer worry and screening (23). We found that a higher cancer worry score was associated with completion of colonoscopy, which suggests that it may be a motivating factor to attend surveillance testing in higher risk populations. Cancer worry has been shown to predict perceived comparative and absolute colon cancer risk (24). For patients with a history of adenoma, such as those included in this study, cancer worry may be a reflection of higher perceived risk for developing colon cancer compared with other populations. In addition, patients who perceived more benefits of follow-up colonoscopy were more likely to complete screening. This finding is consistent with several studies that have found perceived benefits or outcome expectations to be positively associated with colonoscopy use, including studies of high-risk populations (e.g., first degree relative with colorectal cancer) $(23,25)$.

Participants in our study who reported a greater number of barriers to screening were less likely to complete follow-up colonoscopy. In studies of average risk colorectal cancer screening, barriers 
Table 2. Multivariable logistic regression of factors associated with attendance at follow-up colonoscopy $(n=204)$

\begin{tabular}{lccc} 
& \multicolumn{3}{c}{ Multivariable model $^{\mathbf{a}}$} \\
\cline { 2 - 4 } Variable & $\boldsymbol{n}$ & OR & $\mathbf{9 5 \%} \mathbf{C l}$ \\
Barriers & 199 & 0.49 & $0.28-0.88$ \\
Benefits & 202 & 2.37 & $1.04-5.41$ \\
Social deprivation index & & & \\
$\quad 0$ & 158 & REF & \\
1 & 33 & 0.90 & $0.39-2.11$ \\
$\geq 2$ & 11 & 0.09 & $0.01-0.76$ \\
Hospitalized & & & \\
$\quad$ No & 172 & REF & \\
$\quad$ Once or more & 32 & 0.42 & $0.17-1.03$ \\
Cancer worry & 199 & 1.73 & $1.07-2.79$
\end{tabular}

95\% Cl, 95\% confidence interval; OR, odds ratio; REF, referent.

NOTE: overall mean (s.d.) for psychosocial scales: Barriers 2.07 (0.648),

Benefits 3.44 (0.395), and Cancer worry 2.49 (0.711). Missing values ranged

from 1 (family history, marital status) to 8 (intention, perceived susceptibility).

Participants with missing data on variables included in the multivariable model

were excluded from the analysis. The reduced multivariable model consists of

194 observations (97 attender, 97 non-attender).

aThe following variables were entered into a multivariable model: age, race, marital status, education, intention, barriers, benefits, self-efficacy, social deprivation, comorbidity, family history of colorectal cancer, previous inpatient hospitalizations, smoking status, self-rated health, functional status, cancer worry, and perceived susceptibility. Wald's selection of $P=0.10$ was then used as removal probability until a reduced model for the data was obtained. Reduced multivariable model shown in table. All variables adjusted for other variables in the model.

have an important role in adherence to screening recommendations among individuals who have never been screened, as well as those who are overdue to be screened (25-27). Research suggests that there are important differences in how screening barriers are conceptualized based on the timing of the most recent screening (15). Individuals who are up-to-date with screening tend to report procedural barriers (e.g., dislike of prep solution, discomfort during the exam), whereas those overdue to be screened often cite facilitation barriers (e.g., time, difficulty making an appointment) (28-30). Although the barrier scale used in this study contained both procedure- and facilitation-oriented items, non-attenders scored higher on items related to facilitation, such as transportation, insurance, and scheduling. Similarly, a higher social deprivation score (i.e., no car ownership, no home ownership, and/or low education) was associated with lower odds of screening completion. Few attenders scored above zero on the social deprivation scale, which may help explain the magnitude of the OR and the wide confidence limits. However, taken collectively with the findings on barriers, the results suggest that reducing or eliminating structural barriers by improving access to screening may increase attendance at surveillance colonoscopy.

Despite our initial expectation, patient comorbidity and functional status were not related to screening completion in the
Table 3. Mean score for items on the perceived barriers scale among attenders $(n=100)$ and non-attenders $(n=104)$

\begin{tabular}{|c|c|c|}
\hline & Attenders & Non-attenders \\
\hline & mean (s.d.) & mean (s.d.) \\
\hline $\begin{array}{l}\text { I do not think that a colonoscopy is } \\
\text { needed }\end{array}$ & $1.4(0.99)$ & $1.7(1.24)$ \\
\hline $\begin{array}{l}\text { I am concerned about the cost of a } \\
\text { colonoscopy* }\end{array}$ & $2.6(1.37)$ & $3.0(1.62)$ \\
\hline $\begin{array}{l}\text { I am concerned about the amount of the } \\
\text { medical insurance co-pay* }\end{array}$ & $2.4(1.34)$ & $2.8(2.82)$ \\
\hline $\begin{array}{l}\text { I am concerned about having discomfort } \\
\text { during the procedure }\end{array}$ & $2.2(1.37)$ & $1.9(1.25)$ \\
\hline $\begin{array}{l}\text { I have trouble with transportation to or } \\
\text { from the test* }\end{array}$ & $1.5(1.14)$ & 1.9 (1.39) \\
\hline $\begin{array}{l}\text { I do not want to undergo the prep before } \\
\text { the test }\end{array}$ & $3.2(1.53)$ & $3.3(1.59)$ \\
\hline $\begin{array}{l}\text { I do not have anyone to help me after } \\
\text { the test* }\end{array}$ & $1.4(0.95)$ & $1.8(1.36)$ \\
\hline I do not have time to get a colonoscopy* & $1.4(0.73)$ & $1.8(1.20)$ \\
\hline $\begin{array}{l}\text { I am concerned about medical complica- } \\
\text { tions of the colonoscopy }\end{array}$ & $2.2(1.26)$ & $2.1(1.18)$ \\
\hline $\begin{array}{l}\text { I do not think my health insurance covers } \\
\text { a colonoscopy* }\end{array}$ & $1.2(0.49)$ & $1.5(1.00)$ \\
\hline
\end{tabular}

multivariable model. Comorbidity and other similar measures (e.g., health rating) appeared important in the univariable analysis, but only previous inpatient hospitalizations $(\geq 1)$ remained in the reduced model. Although we did not have information on the reason for hospitalization, it may be that this measure best captures the most critical aspects of health status. Patient comorbidity was measured as a composite index of diabetes, chronic obstructive pulmonary disease/emphysema, and history of other cancers. Because these comorbid conditions are relatively well managed, they may not impede the ability to get screened. More debilitating comorbidities, such as those that require hospitalization, may limit the use of colonoscopy. Future researchers may wish to examine how the various aspects of health status prevent or promote the use of surveillance colonoscopy.

Our results also highlight the need for multilevel interventions that facilitate appropriate use of surveillance colonoscopy. Patient navigation has gained attention as an effective intervention strategy to promote colorectal screening in healthcare and community settings by addressing patient reported barriers to screening (e.g., transportation), and they may be even more effective in facilitating surveillance. Although patient navigation was developed to reduce disparities related to late-stage cancer diagnoses, it has more recently been associated with improvements across the cancer care continuum $(31,32)$. Navigation varies with respect to intervention dose, frequency of contact, and duration; however, most interventions are delivered primarily via telephone 
or a combination of telephone and mail (31). Several intervention studies of average risk colorectal cancer screening have used aspects of patient navigation to increase the uptake of colonoscopy or fecal occult blood test (33-35). These studies, which often include nurse navigators who call patients to discuss any concerns or barriers to screening and encourage completion of the patient's preferred screening tests, have shown significant improvements in screening adherence compared with standard care. By addressing patient and system barriers to accessing screening, navigation may also be a useful strategy to encourage completion of followup colonoscopy at recommended intervals in populations at increased risk for colorectal cancer.

A strength of our study is the use of previously validated measures of colorectal cancer screening benefits, barriers, self-efficacy, cancer worry, and perceived susceptibility (12-17). Many of these measures were developed for average risk screening, but our study demonstrates they can also be appropriately used in previously screened, higher risk populations. We also used validated measures of comorbidity (19), functional status (20,21), and social deprivation (18). Using valid and reliable measures improves the application of psychosocial constructs to interventions that successfully increase adherence to colonoscopy.

The study population was mostly white, highly educated patients who were part of a healthcare system, and was limited to English speaking individuals. Participants who could not be contacted via mail and/or telephone were not included in the study. Because study participants were required to have a deliverable address and agree to be interviewed, the study population may have had more healthcare resources compared with the general population. Colorectal cancer screening rates remain low among non-Whites and those with less education, living in rural vs. urban areas, and without a regular source of healthcare or medical insurance (36). There may be other factors associated with the use of surveillance colonoscopy in more diverse samples who may be at most risk of nonattendance. In addition, we could not identify patients who had moved away from the healthcare system after their index colonoscopy. It is unknown whether these patients completed a followup exam or if and how changes in medical care influenced receipt of follow-up colonoscopy at recommended intervals. Our study was cross-sectional and could only establish associations in a small sample of patients. Although we have provided new evidence on the importance of multilevel factors associated with surveillance colonoscopy, the small sample size may have affected the magnitude and precision of our estimates. Further, our results cannot explain how these factors affect screening behavior. Understanding the underlying causal mechanisms that link these factors to patient use of colonoscopy will allow researchers to make informed decisions about how and when to intervene.

Previous research has cited overuse of colonoscopy as a growing problem (37-39); however, our results demonstrate that there are a substantial proportion of patients who do not complete follow-up colonoscopy. On the basis of our conceptual framework, predisposing characteristics, enabling factors, and patient need emerged as important factors that contribute to the underuse of surveillance colonoscopy. Identifying these factors is a first step toward developing interventions that promote appropriate use of colonoscopy, which may ultimately improve the quality of colorectal cancer surveillance and reduce the cost and burden of cancer morbidity and mortality.

\section{CONFLICT OF INTEREST}

Guarantor of the article: Caitlin C. Murphy, MPH.

Specific author contributions: Caitlin C. Murphy: analysis and interpretation of data, drafting of the manuscript, statistical analysis; Carmen L. Lewis: study concept and design, critical revision of manuscript; Carol E. Golin: study concept and design, critical revision of manuscript; Robert S. Sandler: study concept and design, critical revision of manuscript, study supervision.

Financial support: National Institutes of Health grants $\mathrm{t} 32$ DK07634, P30 DK034987, and R01 CA55684, and UNC Lineberger Comprehensive Cancer Center. There was no financial or editorial assistance received to support the preparation of the article.

Potential competing interests: None.

\section{REFERENCES}

1. Siegel R, Desantis C, Jemal A. Colorectal cancer statistics, 2014. CA: Cancer J Clin 2014;64:104-17.

2. Edwards BK, Ward E, Kohler BA et al. Annual report to the nation on the status of cancer, 1975-2006, featuring colorectal cancer trends and impact of interventions (risk factors, screening, and treatment) to reduce future rates. Cancer 2010;116:544-73.

3. Klabunde CN, Cronin KA, Breen $\mathrm{N}$ et al. Trends in colorectal cancer test use among vulnerable populations in the United States. Cancer Epidemiol Biomarkers Prev 2011;20:1611-21.

4. Seeff LC, Nadel MR, Klabunde CN et al. Patterns and predictors of colorectal cancer test use in the adult US population. Cancer 2004;100:2093-103.

5. Peery AF, Dellon ES, Lund J et al. Burden of gastrointestinal disease in the United States: 2012 update. Gastroenterol 2012;143:1179-87.e1-3.

6. Lieberman DA, Rex DK, Winawer SJ et al. Guidelines for colonoscopy surveillance after screening and polypectomy: a consensus update by the US Multi-Society Task Force on Colorectal Cancer. Gastroenterol 2012;143:844-57.

7. Schoen RE, Pinsky PF, Weissfeld JL et al. Utilization of surveillance colonoscopy in community practice. Gastroenterol 2010;138:73-81

8. Andersen RM. Revisiting the behavioral model and access to medical care does it matter? J Health Soc Behav 1995;36:1-10.

9. Vernon SW, Bartholomew LK, McQueen A et al. A randomized controlled trial of a tailored interactive computer-delivered intervention to promote colorectal cancer screening: sometimes more is just the same. Ann Behav Med 2011;41:284-99.

10. Tilley BC, Vernon SW, Myers R et al. The Next Step Trial: impact of a worksite colorectal cancer screening promotion program. Prev Med 1999;28:276-83.

11. Green BB, Wang CY, Horner K et al. Systems of support to increase colorectal cancer screening and follow-up rates (SOS): design, challenges, and baseline characteristics of trial participants. Contemp Clin Trials 2010;31:589-603

12. Vernon SW, Myers RE, Tilley BC. Development and validation of an instrument to measure factors related to colorectal cancer screening adherence. Cancer Epidemiol Biomarkers Prev 1997;6:825-32.

13. Tiro JA, Vernon SW, Hyslop T et al. Factorial validity and invariance of a survey measuring psychosocial correlates of colorectal cancer screening among African Americans and Caucasians. Cancer Epidemiol Biomarkers Prev 2005;14:2855-61.

14. McQueen A, Tiro JA, Vernon SW. Construct validity and invariance of four factors associated with colorectal cancer screening across gender, race, and prior screening. Cancer Epidemiol Biomarkers Prev 2008;17:2231-7.

15. Murphy CC, McQueen A, Bartholomew LK et al. Factorial validity and invariance of four psychosocial constructs of colorectal cancer screening: does screening experience matter? Cancer Epidemiol Biomarkers Prev 2013;22:2295-302. 
16. Ritvo P, Myers R, Del Giudice ML et al. Factorial validity and invariance of a survey measuring psychosocial correlates of colorectal cancer screening in Ontario, Canada-a replication study. Cancer Epidemiol Biomarkers Prev 2008;17:3279-83.

17. Rawl S, Champion V, Menon U et al. Validation of scales to measure benefits of and barriers to colorectal cancer screening. J Pyschosocial Oncol 2001;19:47-63

18. Power E, Van Jaarsveld CH, McCaffery K et al. Understanding intentions and action in colorectal cancer screening. Ann Behav Med 2008;35:285-94.

19. Katz JN, Chang LC, Sangha O et al. Can comorbidity be measured by questionnaire rather than medical record review? Med Care 1996;34:73-84

20. Katz S. Assessing self-maintenance: activities of daily living, mobility, and instrumental activities of daily living. J Am Geriatr Soc 1983;31:721-7.

21. Ware J, Jr., Kosinski M, Keller SD. A 12-Item Short-Form Health Survey: construction of scales and preliminary tests of reliability and validity. Med Care 1996;34:220-33.

22. Hay JL, Buckley TR, Ostroff JS. The role of cancer worry in cancer screening: a theoretical and empirical review of the literature. Psychooncology 2005; 14:517-34.

23. McQueen A, Vernon SW, Myers RE et al. Correlates and predictors of colorectal cancer screening among male automotive workers. Cancer Epidemiol Biomarkers Prev 2007;16:500-9.

24. Hay J, Coups E, Ford J. Predictors of perceived risk for colon cancer in a national probability sample in the United States. J Health Comm 2006;11(Suppl 1):71-92

25. Kiviniemi MT, Bennett A, Zaiter M et al. Individual-level factors in colorectal cancer screening: a review of the literature on the relation of individuallevel health behavior constructs and screening behavior. Psychooncology 2011;20:1023-33.

26. Janz NK, Wren PA, Schottenfeld D et al. Colorectal cancer screening attitudes and behavior: a population-based study. Prev Med 2003;37:627-34

27. Greiner KA, James AS, Born W et al. Predictors of fecal occult blood test (FOBT) completion among low-income adults. Prev Med 2005;41:676-84.

28. Jones RM, Devers KJ, Kuzel AJ et al. Patient-reported barriers to colorectal cancer screening: a mixed-methods analysis. Am J Prev Med 2010;38:508-16.
29. Jones RM, Woolf SH, Cunningham TD et al. The relative importance of patient-reported barriers to colorectal cancer screening. Am J Prev Med 2010;38:499-507

30. Medina GG, McQueen A, Greisinger AJ et al. What would make getting colorectal cancer screening easier? Perspectives from screeners and nonscreeners. Gastroenterol Res Pract 2012;2012:895807.

31. Paskett ED, Harrop JP, Wells KJ. Patient navigation: an update on the state of the science. CA: Cnacer J Clin 2011;61:237-49.

32. Wells KJ, Battaglia TA, Dudley DJ et al. Patient navigation: state of the art or is it science? Cancer 2008;113:1999-2010

33. Green BB, Wang CY, Anderson ML et al. An automated intervention with stepped increases in support to increase uptake of colorectal cancer screening: a randomized trial. Ann Intern Med 2013;158:301-11.

34. Myers RE, Bittner-Fagan $\mathrm{H}$, Daskalakis $\mathrm{C}$ et al. A randomized controlled trial of a tailored navigation and a standard intervention in colorectal cancer screening. Cancer Epidemiol Biomarkers Prev 2013;22:109-17.

35. Lasser KE, Murillo J, Lisboa S et al. Colorectal cancer screening among ethnically diverse, low-income patients: a randomized controlled trial. Arch Intern Med 2011;171:906-12.

36. Shapiro JA, Klabunde CN, Thompson TD et al. Patterns of colorectal cancer test use, including CT colonography, in the 2010 National Health Interview Survey. Cancer Epidemiol Biomarkers Prev 2012;21:895-904

37. Goodwin JS, Singh A, Reddy N et al. Overuse of screening colonoscopy in the Medicare population. Arch Intern Med 2011;171:1335-43.

38. Mysliwiec PA, Brown ML, Klabunde CN et al. Are physicians doing too much colonoscopy? A national survey of colorectal surveillance after polypectomy. Ann Intern Med 2004;141:264-71.

39. Partin MR, Powell AA, Bangerter A et al. Levels and variation in overuse of fecal occult blood testing in the Veterans Health Administration. J Gen Intern Med 2012;27:1618-25.

This work is licensed under a Creative Commons Attribution-NonCommercial-NoDerivs 3.0 Unported License. To view a copy of this license, visit http://creativecommons. org/licenses/by-nc-nd/3.0/

\section{APPENDIX 1}

Barriers (10 items)

- I do not think that a colonoscopy is needed

- I am concerned about the cost of a colonoscopy

- I am concerned about the amount of the medical insurance co-pay

- I am concerned about having discomfort during the procedure

- I have trouble with transportation to or from the test

- I do not want to undergo the prep before the test

- I do not have anyone to help me after the test

- I do not have time to get a colonoscopy

- I am concerned about medical complications of the colonoscopy

- I do not think my health insurance covers a colonoscopy

Benefits (7 items)

- Doing colorectal cancer screening makes sense to me

- I think the benefits of colorectal screening outweigh any difficulty I might have in going through the test

- Going through colorectal screening is an important thing for me to do

- I believe that colorectal screening can help protect my health

- I believe that if I had a normal screening test result, I wouldn't have to worry about developing colorectal cancer

- I think when colorectal polyps are found and removed, colorectal cancer can be prevented

- I believe when colorectal cancer is found early, it can be cured 
Self-efficacy (4 items)

- Arranging my schedule to go through colorectal screening is an easy thing to do

- Finding time to go through colorectal screening would be difficult for me to do*

- Going through colorectal screening would be difficult for me to do*

- I think going through colorectal screening would be an easy thing for me to do

Perceived susceptibility ( 3 items)

- I think that it is very likely that I will develop colorectal cancer or polyps

- I believe that the chance that I will develop colorectal polyps is high

- I believe that the chance I might develop colorectal cancer is high

Cancer worry (4 items)

- I am afraid of an abnormal screening test result

- I am worried that screening will show that I have colorectal cancer or polyps

- I am bothered by the possibility that screening might be physically uncomfortable

- I am afraid of having colon cancer

Social deprivation index

- Do you own a car?

- Do you own the home (place) where you are living now?

- Did you pass high school or college-based exams? (derived from education variable by categorizing less than high school vs. all others)

Instrumental activities of daily living

- Because of a physical, mental, or emotional problem, do you need the help of other persons in handling routine needs such as everyday household chores, doing necessary business, shopping, or getting around for other purposes?

- By yourself, without using any special equipment, how difficult is it for you to walk a quarter of a mile (about 3 city blocks)?

${ }^{\star}$ Indicates item was reverse scored 